\title{
Analysing the Low Quality of the Data in Lighting Control Systems
}

\author{
Jose R. Villar ${ }^{1}$, Enrique de la $\mathrm{Cal}^{1}$, Javier Sedano ${ }^{2}$, and Marco \\ García-Tamargo $^{1}$ \\ 1 Computer Science Department, University of Oviedo, Campus de Viesques s/n \\ 33204 Gijón (SPAIN) \{villarjose, delacal, marco\}@uniovi.es \\ 2 Instituto Tecnológico de Castilla y León, Lopez Bravo 70, Pol. Ind. Villalonquéjar \\ 09001 Burgos (SPAIN) javier.sedano@itcl.es *
}

\begin{abstract}
Energy efficiency represents one of the main challenges in the engineering field, i.e., by means of decreasing the energy consumption due to a better design minimising the energy losses. This is particularly true in real world processes in the industry or in business, where the elements involved generate data full of noise and biases. In other fields as lighting control systems, the emergence of new technologies, as the Ambient Intelligence can be, degrades the quality data introducing linguistic values. The presence of low quality data in Lighting Control Systems is introduced through an experimentation step, in order to realise the improvement in energy efficiency that its of managing could afford. In this contribution we propose, as a future work, the use of the novel genetic fuzzy system approach to obtain classifiers and models able to deal with the above mentioned problems.
\end{abstract}

\section{Introduction}

Energy Efficiency represents a big challange in different engineering fields as electric energy distribution [11], efficient design and operation [9], modeling and simulation [3], etc. In general, multi-agent architecture and the distribution among the intelligent devices of the control and the optimisation of the decisions may improve the energy efficiency [18]. In what follows, the field of lighting control systems will be analysed for the sake of simplicity, although the main conclusions can be extended to any other area.

In a lighting control system (see Fig. 1), the lighting system controller is the software responsible for co-ordinating the different islands and for integrating the information from the Building Management Systems (BMS). In each island, a controller establishes the operation conditions of all the controlled ballasts according to the sensor measurements and the operation conditions given by the lighting system controller.

*This research work is been funded by Gonzalez Soriano, S.A. by means of the the CN-08-028-IE07-60 FICYT research project and by Spanish M. of Science and Technology, under the grant TIN2008-06681-C06-04. 




Fig. 1. The schema of a lighting control system. Each island includes a closed loop controller with the controlled gears, the luminosity sensors, the presence sensors, etc. The lighting system controller is the responsible of co-ordinating the islands.

The lighting control systems have been studied in depth: simulation issues [3], sensor processing and data improvement [4], the effect of daylight in the energy efficiency [7]. Moreover, the improvement in the energy efficiency and its measurement have been analysed in $[7,9]$.

Nevertheless, the meta-information in the data gathered from processes is rarely used, and it is mainly related to non stochastic noise. This meta-information related with the low quality data can also be due to the precision of the sensors and to the emergence of new technologies such as Ambient Intelligence and the user profiles. In our opinion, the use of Genetic Fuzzy Systems (GFS) could improve the issues related with energy sharing and efficiency in distributed systems. We propose using the GFS able to deal with the meta-information to achieve better energy efficiency results.

In this research we show how the uncertainty in real world problems can be observed, specifically, in lighting systems. We propose the use of a novel method for learning GFS with low quality data for improving the energy efficiency in distributed systems taking advantage of the meta-data due to low quality data. The remainder of this manuscript is as follows. Firstly, a review of the literature concerned with considering the low quality data in modeling and in designing indexes is shown. Then, it will be shown the uncertainties in real world problems like the simulation of lighting control systems. Finally, some conclusions in how to manage such low quality data are presented.

\section{Issues in low quality data management}

The need for algorithms able to face low quality data is a well-known fact in the literature. Several studies have presented the decrease in the performance of crisp algorithms as uncertainty in data increases [5].

On the other hand, [10] analyses the complexity nature of the data sets in order to choose the better Fuzzy Rule Based System. Several measures are proposed to deal with the complexity of the data sets and the Ishibuchi fuzzy hybrid 
genetic machine learning method is used to test the validity of the measures. This research also concludes in the need to extend the proposed measures to deal with low quality data.

With low quality data we refer to the data sampled in presence of non stochastic noise or obtained with imprecise sensors. It is worth noting that all the sensors and industrial instrumentation can be regarded as low quality data. In our opinion, one of the most successful researches in soft computing dealing with low quality data is detailed in $[2,13]$. In these works the mathematical basis for designing vague data awareness genetic fuzzy systems -both classifiers and modelsis shown. The low quality data are assumed as fuzzy data, where each $\alpha$-cut represents an interval value for each data.

Finally, it is worth pointing out that the fitness functions to train classifiers and models are also fuzzy valued functions when faced with low quality data. Hence the learning algorithms should be adapted to such fitness functions [16]. The ideas and principles previously shown have been used in several applications with low quality data, with both realistic and real world data sets. $[14,15,19]$.

\section{Low quality data in lighting systems and the energy efficiency}

Lighting control systems aim to set the electric power consumption for the ballast in the installation so the luminance accomplishes with the regulations. In such systems, the luminance is measure through light sensors. Variables as the presence of inhabitants are also used in lighting control systems. Even though there are more variables, the relevance of the former is higher as it is used as the feedback in the lighting control loop. Nevertheless, the output of such sensors is highly dependant of the sunlight, the magnitude varies from one sensor to other, the repeatability is a compromise, etc. Consequently, the output of the sensors is usually filtered and then used as the feedback of the control loop, always as a crisp value.

Simulation of lighting systems has been widely studied, mainly to improve the energy efficiency $[3,7]$. A lighting system simulation needs to simulate the light measured in a room when a total electric power is applied for lighting. A simulation will use models to estimate the response of the light sensors. The main objective in simulation is to set and tune PID controllers for light control systems.

As before, the measurements from light sensors are considered crisp values, and so the inputs and the outputs of the light sensor models. To our knowledge, no model has been obtained including the meta-information due to low quality data and, thus, the effect of the daylight and other variables are introduced artificially -i.e., by considering such information within the input data set.

Let us consider one simple case. Let us suppose the simulation of the lighting system shown in Fig. 2, where there is a simple room with one light sensor installed and the light gears accomplishing the regulations. Where to fix the light sensor is of great significance as the shorter the distance from the light sensor to 
the windows the higher the daylight influence in the light measurements. On the other hand, the daylight should be estimated from the inner light sensors when no daylight sensors are available.

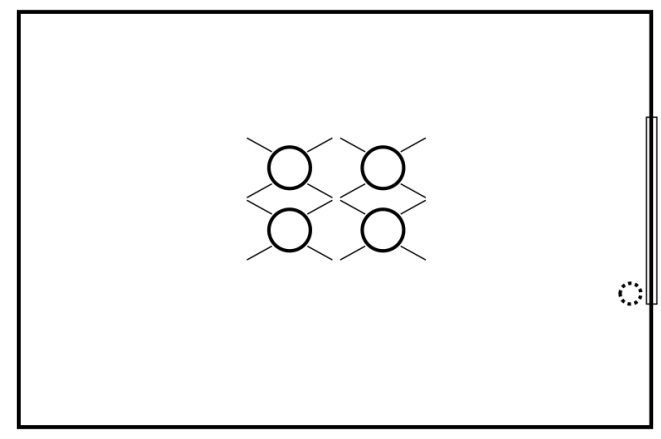

Fig. 2. The lighting system to simulate. Different places for the light sensor are proposed. The light measured will differ from one case to another.

Let us suppose the light sensor installed next to the window, with the light sensor being a LDR (light dependant resistor). Let us also consider a lighting control system that allows regulations on the electric power for lighting, so it is possible to introduce several steps, say $0 \%, 33 \%, 66 \%$ and $100 \%$ of the total power installed. Finally, there was a blind that could be opened or closed. In this scenario, several experiments were carried out. In all of them, the controlled variable was the percentage of electric power for lighting and the output from the light sensor (as a voltage value) was sampled.

The first experiment was the step response increasing and decreasing the controlled variable with the blind closed, measuring the light sensor output. This experiment was carried twice for each sensor, and repeated for five different sensors. In Fig. 3 the results are presented. As can be seen, the measurements are highly dependant of the sensor itself, but also the hysteresis behaviour can be perceived. In all the figures normalised values are presented for the sake of clearness.

Anyway, this is a measure linear with the resistance of the LDR, but what about the luminance? To calculate the lluminance $R=C_{0} \cdot L^{-\gamma}$ applies, where $\mathrm{R}$ is the measured resistance, $C_{0}$ and $\gamma$ are constants for each LDR. LDR's are characterised with a range of possible values of resistance for a luminance of 10 lux $\left(R_{10}\right)$. In case of the ones used in the experiments, $R_{10}$ varies from $[8,20] k \Omega$ and $\gamma$ is 0.85 , typically. Thus, given a sensor, a minimum and a maximum of the luminance can be obtained if a resistance value is measure (see Fig. 4). And for the up/down step responses there is a wide margin of possible values of the luminance as shown in Fig. 5. 




Fig. 3. The normalised output of the different light sensors. The up/down cycle was repeated twice for each one, so the dashed lines corresponds to the second run. Ten samples were consider for each step. No transient was considered, the sample rate was 1 sample/second.

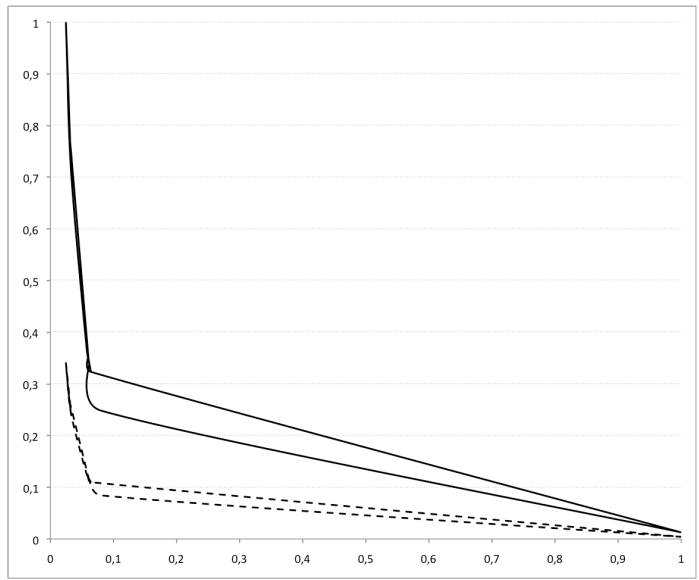

Fig. 4. The resistance against the luminance for the minimum and maximum bounds, both variables in percentage of their maximum value. A measurement of resistance corresponds with an interval of luminance. I.e., if $r=0.6$ induces a luminance in the range $[\sim 0.04, \sim 0.15] \%$, where the hysteresis does not allow to establish the exact values of luminance. 


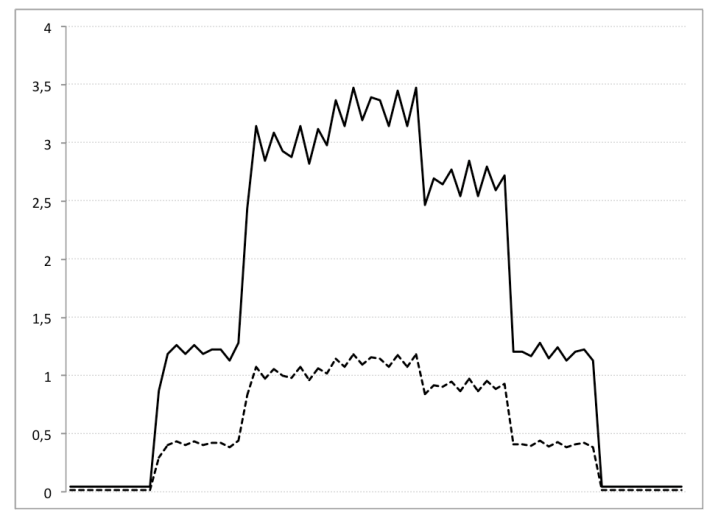

Fig. 5. The minimum and maximum values of luminance for the step responses. It can be seen that for the same power level different luminance values can be calculated using the sensor datasheet. Again, it can be seen the response lacks of hysteresis.

The second experiment includes the opening of the blind. Three positions were consider: totally close, half open and totally open. With the same characteristics as in the first experiment, the step responses for the up/down sequence for one of the sensors is shown in Fig. 6 and Fig. 7.

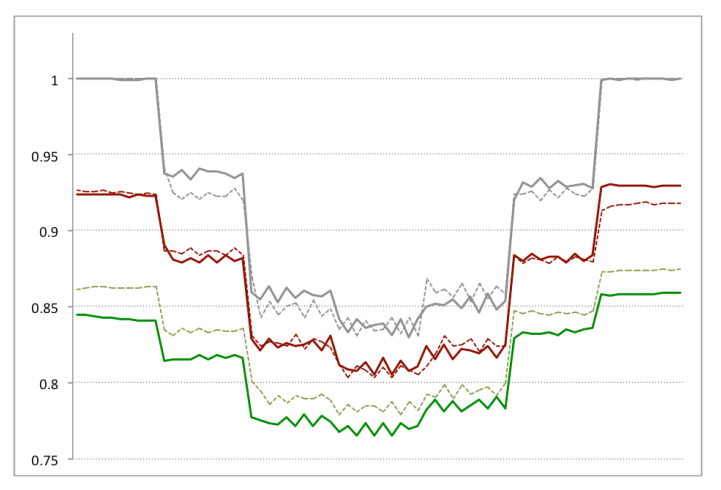

Fig. 6. The normalised output of the light sensors for different blind positions. The up/down cycle was reapeted twice for each one, so the dashed lines corresponds to the second run. Ten samples were consider for each step. No transient was considered, the sample rate was 1 sample/second.

As can be seen, there is an evidence that light controllers can not be optimum, thus the energy efficiency in lighting control systems can be improved if such low quality data is considered. Specifically, the use of the methodology using classical control theory proposed by different authors as [3] may not be the best choose. In our opinion, the use of GFS to obtain models for simulation of lighting systems would help in the integration of the meta-information. The use of GFS allows determining behaviour laws and interpretability of the phenomena. Moreover, if 
low quality data is included in obtaining the models of the sensors the controllers would be robust to such variability.

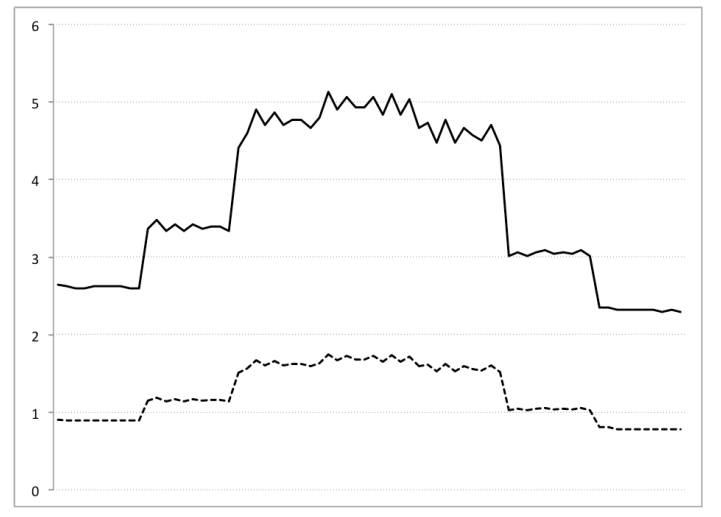

Fig. 7. The minimum and maximum values of luminance for the step responses when the blind is totally opened for a stormy weather day.

\section{Conclusions}

Improving the energy efficiency represents a challenge in the real world applications, especially distributed systems within building management systems. The higher the human interaction in field the higher relevance of intelligent techniques that consider the meta-information and interpretability. Meta-information refers to the information that is present in a process but rarely considered, such as the data non-stochastic noise or the sensor precision and calibration, but also the ambiguity in the linguistic and crisp data. Meta-information can be presented as low quality data.

GFS have been found valid in energy efficiency, but have also been extended to manage interval and fuzzy data. Interval and fuzzy data are mechanisms to represent the low quality data. In this work the extended GFS to manage fuzzy data is proposed to be used in the energy efficiency improvement. To illustrate the idea, the uncertainty in the luminance measurements from light sensors is analysed. We expect that fuzzy data awareness GFS will outperform the modeling and simulation process, so better controllers can be obtained.

\section{References}

1. Bernal-Agustín, J. L. and Dufo-López, R.: Techno-economical optimization of the production of hydrogen from PV-Wind systems connected to the electrical grid. Renewable Energy 35(4), 747-758 (2010)

2. Couso, I. and Sánchez, L.: Higher order models for fuzzy random variables. Fuzzy Sets and Systems 159, 237-258 (2008) 
3. de Keyser, R. and Ionescu, C.: Modelling and simulation of a lighting control system. Simulation Modelling Practice and Theory (2009) DOI: 10.1016/j.simpat.2009.10.003

4. Doulos, L., Tsangrassoulis, A. and Topalis, F. V.: The role of spectral response of photosensors in daylight responsive systems. Energy and Buildings 40(4), 588-599 (2008)

5. Folleco, A. A., Khoshgoftaar, T. M., Van Hulse, J. and Napolitano, A.: Identifying Learners Robust to Low Quality Data. Informatica 33, 245-259 (2009)

6. Gligor, A., Grif, H. and Oltean, S.: Considerations on an Intelligent Buildings Management System for an Optimized Energy Consumption. Proceedings of the IEEE Conference on Automation, Quality and Testing, Robotics (2006)

7. Hviid, C. A., Nielsen, T. R. and Svendsen, S.: Simple tool to evaluate the impact of daylight on building energy consumption. Solar Energy (2009) DOI: 10.1016/j.solener.2008.03.001

8. Houwing, M., Ajah, A. N., Heijnen, P. W., Bouwmans, I. and Herder, P. M.: Uncertainties in the design and operation of distributed energy resources: The case of micro-CHP systems. Energy 33(10), 1518-1536 (2008)

9. Li, D. H. W., Cheung, K. L., Wong, S. L. and Lam, T. N. T.: An analysis of energy-efficient light fittings and lighting controls. Applied Energy 87(2), 558$567(2010)$

10. Luengo, J. and Herrera, F.: Domains of competence of fuzzy rule based classification systems with data complexity measures: A case of study using a fuzzy hybrid genetic based machine learning method. Fuzzy Sets and Systems 161, 3-19 (2010)

11. Martín, J. A. and Gil, A. J.: A new heuristic approach for distribution systems loss reduction. Electric Power Systems Research 78(11), 1953-1958 (2008)

12. Qiao, B., Liu, K. and Guy, C.: A Multi-Agent System for Building Control. IAT '06: Proceedings of the IEEE/WIC/ACM international conference on Intelligent Agent Technology, IEEE Computer Society 653-659 (2006)

13. Sánchez, L. and Couso, I.: Advocating the Use of Imprecisely Observed Data in Genetic Fuzzy Systems. IEEE Transactions on Fuzzy Systems 15(4), 551-562 (2007)

14. Sánchez, L. and Otero, J.: Learning Fuzzy Linguistic Models from Low Quality Data by Genetic Algorithms. In Proceedings of the IEEE Internacional Conference on Fuzzy Systems FUZZ-IEEE 2007 (2007)

15. Sánchez, L., Suárez, M. R., Villar, J. R., and Couso, I.: Mutual Information-based Feature Selection and Fuzzy Discretization of Vague Data. Internacional Journal of Approximate Reasoning 49 607-622 (2008)

16. Sánchez, L., Couso, I. and Casillas, J.: Genetic Learning of Fuzzy Rules based on Low Quality Data. Fuzzy Sets and Systems (2009)

17. Villar, J.R., Pérez, R., de la Cal, E. and Sedano, J.: Efficiency in Electrical Heating Systems: An MAS real World Application. Lecture Notes in Computer Science, Proceedings of the 7th International Conference on Practical Applications of Agents and Multi-Agent Systems (PAAMS 2009) 55, 460-469 (2009)

18. Villar, J. R., de la Cal, E., Sedano, J.: A fuzzy logic based efficient energy saving approach for domestic heating systems. Integrated Computer-Aided Engineering 16(2), 151-164 (2007)

19. Villar, J.R., Otero, A., Otero, J. and Sánchez, L.: Taximeter verification with GPS and Soft Computing Techniques. SoftComputing 14(4), 405-418 (2010) 